\title{
躍Irestice
}

\section{Modelagem Descritiva de um Repositório Institucional de Protocolos de Cuidado à Saúde em hospitais universitários brasileiros}

\author{
Descriptive Modeling of an Institutional Repository of Health Care Protocols in Brazilian \\ University Hospitals
}

\author{
Éllen Daiane Biavatti de Oliveira Algeri ${ }^{1}$, Maria Claudia Medeiros Dantas de Rubim \\ Costa $^{2}$, Hertz Wilton de Castro Lins ${ }^{3}$, Rosemary Silva da Silveira ${ }^{4}$
}

\begin{abstract}
Resumo
Introdução: O volume de informação técnico-científica produzida nas instituições de saúde tem crescido exponencialmente; e reuni-las, armazená-las, recuperá-las e facilitar seu acesso torna-se, então, um desafio. O objetivo do trabalho é construir a modelagem descritiva de um Repositório Institucional (RI) de Protocolos de Cuidado a Saúde que possa atender a essa demanda e ainda otimizar os processos de trabalho e melhorar a qualidade assistencial nos hospitais universitários brasileiros. Métodos: Estudo híbrido de produção e pesquisa metodológica de um RI de Protocolos de Cuidado a Saúde permeada por três etapas: Observação, Revisão Integrativa da literatura e construção da Modelagem Descritiva. Resultados: A modelagem apresenta 3 atores de uso: usuários dos sistemas (servidores/residentes multiprofissionais); gestor local e central. Cada protocolo é inserido no RI através de um formulário padrão estruturado com atualização periódica. Após acessar os protocolos, o usuário pode avaliar seu conteúdo, gerando um feedback ao gestor. O RI produzirá relatórios estatísticos subsidiarão a gestão e a educação permanente. Conclusão: A facilidade de acesso à informação possibilita maior adesão e utilização dos protocolos. O que efetiva a padronização frente a diversidades de condutas, diminuindo os eventos adversos, melhorando a qualidade assistencial, além de conferir respaldo jurídico e reduzir custos aos hospitais.
\end{abstract}

Palavras-chave: Gestão da Qualidade em Saúde, Gestão da Informação, Tecnologia Biomédica.

\section{Abstract}

Introduction: The volume of technical-scientific information produced in health institutions has grown exponentially; and collecting, storing, retrieving, and facilitating their access then becomes a challenge. The objective of this work is to construct a descriptive modeling of an Institutional Repository (RI) of Health Care Protocols that can meet this demand and optimize

\footnotetext{
1 Doutoranda em Enfermagem. Universidade Federal do Rio Grande - FURG, Rio Grande (RS). Email: ellenbiavatti@gmail.com

2 Doutora em Enfermagem. Universidade Federal do Rio Grande do Norte - UFRN. Natal (RN). Email: mclaudiadantas@gmail.com

3 Doutor em Engenharia Elétrica e Computação. Universidade Federal do Rio Grande do Norte - UFRN. Email: hertzw@gmail.com

4 Doutora em Enfermagem. Universidade Federal do Rio Grande - FURG, Rio Grande (RS). Email: rosemaryssilveira@gmail.com

Correspondência: Rua General Câmara, № 219. Apartamento 204. Centro. Rio Grande (RS). CEP: 96. 200-320.
}

RE. SAÚD. DIGI. TEC. EDU., Fortaleza, CE, v.4, n. 2, p.84-98, ago./dez. 2019. ISSN: 2525-9563 
work processes and improve the quality of care in Brazilian university hospitals. Methods: Hybrid study of production and methodological research of an IR of Health Care Protocols permeated by three steps: Observation, Integrative Literature Review and construction of Descriptive Modeling. Results: The modeling presents 3 actors of use: system users (multiprofessional servers / residents); local and central manager. Each protocol is entered into $R I$ through a standard structured form with periodic update. After accessing the protocols, the user can evaluate their content, generating feedback to the manager. RI will produce statistical reports that will support management and continuing education. Conclusion: The ease of access to information enables greater adherence and use of protocols. What makes standardization possible in the face of a variety of behaviors, reducing adverse events, improving the quality of care, providing legal support and reducing costs to hospitals.

Keywords: Quality Management in Healthcare, Information Management, Biomedical Technology.

\section{Introdução}

O gerenciamento da qualidade na área da saúde pode ser definido como a procura por resultados satisfatórios ao paciente por meio da melhoria dos processos de trabalho voltados ao cuidado assistencial ${ }^{1}$.

É imprescindível considerar que os serviços prestados no ambiente hospitalar, envolvem o ser humano em uma situação de vulnerabilidade, acometido de enfermidades diversas e exigem prudência para a realização de intervenções assistenciais de forma adequada e uniformizada ${ }^{1}$.

Trata-se, portanto, de estabelecer padrões de serviços baseados na integralidade humana e na patologia associada, enfatizando as evidências científicas. Desta forma se evita condutas mecanicistas com risco de danos ao paciente ${ }^{1,2}$.
Nesse sentido, os protocolos de cuidado à saúde surgem como parte dos instrumentos para qualificar a atenção hospitalar e diminuir a ocorrência de eventos adversos relacionados a conduta clínica e a execução de procedimentos.

Eles são desenvolvidos baseados em evidências científicas e na experiência do corpo clínico e adaptados aos recursos locais disponíveis. Além disso, buscam fornecer um fluxo padronizado para o manejo do paciente em determinada condição clínica e são elaborados por equipes multidisciplinares ${ }^{3}$.

Os protocolos de cuidado a saúde estão cada vez mais presentes no fazer dos profissionais nas instituições por relacionarem-se com a normalização de ações e registros e à economia de tempo durante a prestação do cuidado. Tais artifícios ganham destaque na produção de saúde pela necessidade de realização de ações padronizadas com qualidade e agilidade ${ }^{4}$. 
Neste contexto, se inserem os Hospitais Universitários (HUs), que são entendidos como centros de formação de recursos humanos e de desenvolvimento de tecnologias para a área de saúde. Ademais, prestam serviços à população, elaboram protocolos técnicos para o manejo de diversas patologias e oferecem programas de educação continuada ${ }^{5}$.

Destarte, o volume de informação técnico-científica produzido nas instituições de saúde, principalmente nos hospitais universitários federais, tem crescido exponencialmente, resultado do avanço das Tecnologias de Informação e Comunicação (TICs). Desta forma, reunir as informações geradas no âmbito institucional, armazená-las e facilitar o acesso dessas torna-se, então, um desafio ${ }^{6}$.

Nessa perspectiva, o Repositório Institucional (RI) de Protocolos de cuidado a Saúde é um recurso que vem atender a essa demanda. Considerando a informação um patrimônio institucional que é produzida por uma equipe multiprofissional, é imprescindível uma gestão que possibilite seu armazenamento em local único e de fácil acesso.

E ainda, o acesso seguro conferido por uma ferramenta tecnológica de protocolos de cuidado a saúde proporciona uma gestão da informação institucional com redução de custos com cópias impressas sem controle, ganhos de eficiência e amparo legal a administração pública ${ }^{6}$.
O RI possui infraestrutura para preservar memória, salvaguardar informações, armazená-las em local único proporcionando facilidade de acesso e otimização dos processos de trabalho. Esta estratégia possibilita o gerenciamento e a disseminação do conhecimento institucional através de uma abordagem inovadora no compartilhamento de informação e na padronização dos protocolos de cuidado a saúde ${ }^{6}$.

Nesse sentido, o presente artigo, resultado da dissertação do programa de Mestrado Profissional em Gestão e Inovação em Saúde da Universidade Federal do Rio Grande do Norte (UFRN) tem como objetivo construir a modelagem descritiva de um Repositório Institucional que possa armazenar, gerenciar, padronizar e conferir facilidade de acesso aos protocolos de cuidados a saúde. E ainda, reduzir custos, otimizar os processos de trabalho, diminuir os eventos adversos e melhorar a qualidade assistencial nos hospitais universitários brasileiros.

A modelagem do RI foi desenvolvida por uma equipe multiprofissional (área assistencial, gestão e setor de Sistemas de Informação) inserida na rede de hospitais universitários federais, com propósito de construir uma ferramenta tecnológica que pudesse atender às necessidades institucionais, gerando uma mudança real na prática e assistencial e, que não se tornasse obsoleta com o passar do tempo. 


\section{Métodos}

Trata-se de um estudo híbrido de produção tecnológica e pesquisa metodológica de um Repositório Institucional de Protocolos de Cuidado a Saúde que se desenvolveu em três etapas.

\subsection{Observação}

A primeira etapa compreendeu a observação, como estratégia de abranger a perspectiva descritiva e interpretativa de como ocorre o gerenciamento dos protocolos de cuidado a saúde. Ou seja, identificar a maneira como eles são armazenados e disponibilizados a equipe multiprofissional (médicos, enfermeiros, fisioterapeutas, nutricionista, dentre outros) em um Hospital Universitário na região Sul do País.

A partir da observação in loco e da vivência profissional, inferiu-se que os profissionais da equipe multiprofissional estão a mercê de adotar condutas clínicas diversas, uma vez que o fortalecimento da gestão da informação e do conhecimento no Hospital por meio da implementação e disseminação efetiva dos protocolos de cuidado a saúde ainda é incipiente. Além disso, os protocolos existentes encontramse disponíveis na intranet 0 que impossibilita um acesso concomitante na rede de hospitais universitários federais.

Foi possível observar ainda que alguns protocolos estão disponíveis em Portable Document Format (PDF) na área de trabalho dos computadores nos diversos setores do hospital, o que compromete a segurança do protocolo, permitindo a produção de cópias sem controle.

\subsection{Revisão Integrativa da Literatura}

A segunda etapa do percurso metodológico consistiu na realização de uma revisão integrativa da literatura com o objetivo de aprofundamento da temática e fundamentação da importância da proposta. A revisão integrativa foi realizada com base no modelo proposto por Kitchenham 7, por melhor se enquadrar no estudo híbrido, uma vez que é direcionado as pesquisas voltadas a engenharia de software.

Foi realizada a busca nas bases de dados: LILACS, SCIELO e MEDLINE com data limite de julho de 2018 utilizando as seguintes palavras-chaves: Gestão da Qualidade em Saúde; Gestão da Informação; Tecnologia Biomédica. Elas foram combinadas com associações e desfechos de interesse utilizando o termo AND.

É relevante destacar que a busca nas bases de dados foi realizada com data limite de julho de 2018 sem data de início devido a carência das produções científicas que abordassem a temática em seu sentido bem delimitado ${ }^{3}$.

A atividade de seleção dos estudos concentrou-se em avaliar os artigos quanto a sua relevância em relação à pesquisa realizada. O critério de seleção (inclusão e exclusão) destinou-se a identificar os 
estudos que forneciam evidência direta sobre a questão de pesquisa, objetivando reduzir a probabilidade de uma seleção tendenciosa ${ }^{7}$.

Nesse sentido, foram utilizados como critérios de inclusão: trabalhos relevantes que se relacionaram com a pergunta de pesquisa, e seleção de idiomas (inglês, português e espanhol). $\mathrm{E}$ como critérios de exclusão: os artigos duplicados nas bases de dados, artigos indisponíveis na íntegra e aqueles que não respondiam à pergunta da pesquisa. Em conseguinte, obteve-se um fluxo metodológico conforme (Fig. 1 - anexos).

É relevante citar que, além da revisão nos bancos de dados foram utilizados manuais técnicos e resoluções relacionados ao tema e vinculados a Organização Mundial de Saúde (OMS), Ministério da Saúde (MS) e Empresa Brasileira de Serviços Hospitalares (Ebserh).

\subsection{Construção da Modelagem}

\section{Descritiva}

A terceira fase da metodologia consistiu na construção da modelagem descritiva de um repositório institucional, como ferramenta de gestão da qualidade.

O RI permitirá gerenciar, armazenar, padronizar e viabilizar o acesso dos protocolos de cuidado a saúde após a institucionalização das práticas clínicas.
A modelagem descritiva viabiliza criar uma maneira objetiva e compreensível de apresentar as especificidades e funcionalidades de um sistema8 por meio do mapa conceitual, fluxograma de entrada dos protocolos no sistema, diagrama de atores de uso com Unified Modeling Language (UML) ${ }^{1}$ e um formulário estruturado padrão com informações obrigatórias.

Optou-se por esse tipo de modelagem, uma vez que o objetivo foi enfatizar as informações que o RI iria gerenciar e não na arquitetura do software em si. A modelagem foi desenvolvida pensando em um formato em rede, todavia ela pode ser aplicada em um formado local, substituindo o gestor local por unidades/setores e o gestor central por gerência/chefia.

A modelagem foi desenvolvida por uma equipe multiprofissional composta por profissional da área assistencial (uma enfermeira), duas gestoras, sendo uma superintendente de um hospital universitário federal e a outra chefe de Gestão de Cuidados; um programador e técnico em Tecnologia da Informação com vivência no $\mathrm{HU}$. Em seguida, foi desenvolvido um protótipo para validação da modelagem. 


\section{Resultados}

\section{Mapa Mental}

Atualmente, várias técnicas derivadas dos modelos de mapa mental têm sido consideradas como poderosas ferramentas para gerenciar o processo de demonstração dos requisitos em uma modelagem e proporcionar um desenvolvimento ágil ${ }^{9}$.

O mapa mental busca modelar o sistema, informando os pontos principais para gerar um diagrama de casos de uso. Ele permite que o criador do mapa tenha mais liberdade ao expressar suas ideias para desenvolver a modelagem do sistema ${ }^{9}$.

Nesse contexto, optou-se por utilizar características objetivas e cognitivas na descrição da modelagem para atingir o objetivo de transformar ideias em requisitos e ainda, organizá-las de forma que as relações entre os conceitos fossem representadas de forma gráfica através do mapa mental.

Assim, o mapa mental resulta na interpretação do processo onde o fluxo se apresenta de maneira facilmente compreensível (Fig. 2 - anexos).

\section{Fluxograma de entrada dos Protocolos no sistema}

O fluxograma Interfuncional consiste na descrição gráfica de atividades, sendo identificados fluxos de entrada, início e fim das atividades, além de informar quem as executa ${ }^{10}$.
O fluxo de entrada dos protocolos no sistema inicia-se com gestor local (filial) que elabora os protocolos, incorpora-os no sistema e aguarda a liberação do gestor central (sede). Este por sua vez, avalia os protocolos, libera-os ou solicita edição.

Uma vez que os protocolos são liberados pela sede, eles ficam disponíveis para consulta de acordo com as características de cada protocolo. Nesse sentido, o protocolo pode ser padronizado para toda a rede ou mantido em determinada filial (HU) devido as suas especificidades.

A dinâmica do fluxo pode ser visualizada na Fig. 3 (anexos).

A produção e incorporação dos protocolos de cada hospital universitário no repositório são realizadas pelo gestor local, ou seja, em cada filial. É responsabilidade do gestor local ainda, controlar o acesso dos usuários do sistema e revisar os protocolos a cada 2 anos. A data de validade de cada protocolo aparecerá no perfil do gestor para que não haja atraso nas atualizações.

Esses protocolos ficarão armazenados no repositório e serão liberados após análise e aprovação da sede. O gestor central (sede) é responsável pela validação do cadastro dos usuários, controle de acesso, revisão, liberação e padronização dos protocolos nas filiais. Os protocolos, uma vez inseridos e liberados, ficam disponíveis para acesso dos usuários do sistema. 
A proposta de construção da modelagem não visualiza apenas propor um local de armazenamento, mas pretende que ela seja uma ferramenta de gestão da clínica que permita a efetivação dos protocolos de cuidado a saúde. Nesse sentido, após acessar/consultar os protocolos, o usuário poderá estabelecer comentários e avaliar o conteúdo do protocolo (péssimo, ruim, regular, bom, ótimo) para que assim, o gestor obtenha um feedback do usuário em relação a linguagem e adequação do protocolo frente a realidade institucional. A partir desse feedback o gestor pode avaliar se 0 protocolo confere aplicabilidade e está de fato contribuindo para a melhoria da qualidade assistencial.

Além disso, ao final de cada protocolo ficarão disponíveis os links dos conteúdos do referencial bibliográfico com o intuito de estimular o usuário a aprofundar/aperfeiçoar seus conhecimentos acerca do tema do protocolo através de evidências científicas atualizadas.

\section{Diagrama de casos de uso}

O diagrama de casos de uso identifica as funcionalidades que o sistema que está sendo modelado deve oferecer. Esse diagrama objetiva apresentar uma visão externa geral das funcionalidades que o sistema oferecerá aos usuários, sem se preocupar com a maneira como estas funcionalidades serão implementadas ${ }^{11}$.
Cada caso de uso é representado por uma elipse, com uma descrição no seu interior, que identifica uma certa funcionalidade. Toda funcionalidade deve fazer parte de um caso de uso, que é disparado por um ator9. Assim, os atores representam os papéis desempenhados pelos usuários que utilizarão os serviços e funções do sistema ${ }^{11}$.

No RI são identificados 3 atores de uso: usuários do sistema: servidores/residentes multiprofissionais; gestor local (filial); gestor central (sede) evidenciado no diagrama de casos de uso (Fig. 4 - anexos) desenvolvido com através da linguagem UML.

Em relação ao acesso, a proposta consiste na possibilidade de o usuário acessar o RI por meio de um link na área de trabalho dos computadores disponíveis nos Hospitais Universitários. Os servidores/residentes multiprofissionais e gestores terão acesso ao repositório através de um login e senha disponibilizados pelo sistema de autenticação, após realização do cadastro de primeiro acesso.

Esse cadastro é composto por informações pessoais (nome, cadastro de pessoa física - CPF) e profissionais (e-mail institucional; Hospital e unidade de lotação e categoria profissional). As informações profissionais são pré-requisitos para a tela inicial do perfil de cada usuário. Assim quando o usuário acessar o repositório, sua tela inicial será personalizada constando os protocolos de cuidado a 
saúde de sua categoria profissional, do seu Hospital Universitário e setor de atuação.

O desenvolvimento de uma tela inicial personalizada com o perfil do usuário visa otimizar a consulta aos protocolos, uma vez que rotineiramente o usuário utilizará os protocolos vinculados a sua prática profissional. No entanto, o repositório permitirá que cada usuário faça buscas por protocolos de interesses tanto de outros setores do HU de lotação quanto de qualquer $\mathrm{HU}$.

\section{Padronização na inserção dos} protocolos no Repositório Institucional

Cada protocolo será inserido no repositório através de um formulário estruturado de uma maneira didática e sucinta (quadro 1 - anexos), facilitando a compreensão do profissional e agilizando a busca.

A criação de um formulário estruturado com informações obrigatória evita 0 desenvolvimento tanto de protocolos muito sucintos, com falta de informações relevante, quanto de protocolos demasiadamente extensos, com informações desnecessárias. Nos dois casos, há prejuízo nas consultas aos protocolos e consequentemente, na sua aplicabilidade na realidade institucional.

Ademais, quando os protocolos seguem uma estrutura padrão, permite que o usuário encontre as informações de interesse e se habitue a consultar os protocolos, uma vez que esses apresentam-se de uma maneira já conhecida, permitindo uma leitura dinâmica em casos onde a consulta é fundamental, mas requer rapidez.

Além disso, a utilização de protocolos de cuidado a saúde é a maneira mais eficaz de garantir que a equipe multiprofissional conheça cada um dos processos voltados a sua prática diária. $\mathrm{E}$ diante da diversidade de aspectos organizacionais e estruturais, atualizações e rotatividade de servidores reafirma-se a necessidade de se estabelecer um formato padrão com planejamentos direcionados e aplicáveis, porém flexíveis a variedade de protocolos $^{6}$ - operacionais padrões; manuais, fluxos de acessos, dentre outros.

A modelagem do RI propõe, ainda, a produção de dados estatísticos, assim, as atividades que decorrem do funcionamento regular do uso do repositório poderão ser analisadas pelos gestores por meio de relatórios. Os relatórios estatísticos serão discriminados por: Acesso, Categoria Profissional; Protocolo; Setor; Hospital Universitário.

Desse modo, o gestor poderá acompanhar esses relatórios e produzir indicadores que poderão tanto na gestão, quanto nos processos de educação permanente nos hospitais universitários.

Em relação a segurança, para que haja fidedignidade dos dados, é necessário que o $\mathrm{HU}$ faça a análise e validação dos dados cadastrais.

Cabe salientar também que o desenvolvimento do protótipo para validação da modelagem foi encaminhado 
para registro junto ao Núcleo de Inovação Tecnológica (NIT) da Universidade Federal do Rio Grande do Norte e ao Setor Jurídico da Empresa Brasileira de Serviços Hospitalares.

Além disso, a proposta de modelagem e o protótipo de validação vem acompanhada de algumas sugestões de dois trabalhos futuros: gamefication baseado no acesso e um aplicativo de celular a partir da plataforma WEB. O primeiro surge como uma estratégia de encorajar o engajamento dos profissionais a se dedicarem as tarefas organizacionais; enquanto que o segundo, permitiria que os usuários pudessem acessar os protocolos sem depender dos computadores disponíveis do setor que, muitas vezes, se encontram ocupados pela equipe multiprofissional para realizar outras atividades voltados a prática assistencial.

\section{Conclusão}

Uma das características dos hospitais universitários refere-se à rotatividade da equipe multiprofissional e trocas na gestão. Com isso há perdas de conhecimentos e experiências, uma vez que o profissional leva consigo todo 0 aprendizado obtido, gerando prejuízos de tempo e esforço tanto por parte da nova gestão quanto dos trabalhadores remanejados em conhecer as rotinas de trabalho.

A padronização por meio dos protocolos e principalmente seu armazenamento e facilidade de acesso garantem que os conhecimentos fiquem na instituição de saúde e não com profissionais específicos. Nesse sentido o repositório institucional compõe a gestão do conhecimento do hospital.

Ademais, o repositório digital permitirá tanto a gestão dos protocolos de cuidado a saúde de uma unidade hospitalar como de uma rede de hospitais, gerando impacto na gestão da qualidade. A facilidade de acesso à informação aos profissionais de saúde possibilita maior adesão a utilização dos protocolos, efetivando, consequentemente a padronização frente a diversidades de condutas adotadas nos hospitais universitários.

Diante disso, o $\mathrm{RI}$ proporcionará a redução de custos, a otimização do processo de trabalho, a diminuição dos riscos de danos tanto aos pacientes quanto aos profissionais, a melhoria na qualidade assistencial pela mudança real na prática diária, além de conferir respaldo jurídico a instituição hospitalar.

E, ao ser desenvolvida por uma equipe multiprofissional com vivência local, a modelagem do $\mathrm{RI}$ foi adequada $\mathrm{a}$ realidade institucional, respeitando inclusive os fluxos de processos já instituídos quanto aos sistemas de informação existentes. Destarte, a modelagem posposta permite a integração entre gestão da qualidade, tecnologia e assistência 


\section{Referências}

1. Gama ZAS, Saturno PJ. A Segurança do Paciente Inserida na gestão da qualidade dos Serviços de Saúde.

Disponível em:

http://www20.anvisa.gov.br/segurancadop aciente/images/documentos/livros/Livro1Assistencia_Segura.pdf. 1를. ed.; 2013 (acessado em 12 de maio de 2017). 2. WHO, Delivering quality health services. A global imperative for universal health coverage (2018);

http://www.who.int/servicedeliverysafety/q uality-report/publication/en/ (acessado em 06 de julho de 2018.

3. Mourão Netto JJ,_Dias, MAS, Goyanna NF. Uso de instrumentos enquanto Tecnologia para a Saúde. Saúde em Redes. 2016; 2 (1): 65 - 72. Disponível em:

http://revista.redeunida.org.br/ojs/index.ph $\mathrm{p} / \mathrm{rede}-$ unida/article/view/655. Acesso em: jul. 2018. doi:

http://dx.doi.org/10.18310/24464813.2016v2n1p65-72.

4. Mourão Netto JJ,_Dias, MAS, Goyanna NF. Promoção da saúde e a produção de instrumentos para o adolescente: revisão integrativa. Rev. enferm UFPE on line, agosto/2015; 9(Supl.7):9104- 10.

5. Brasil, Ministério da Educação (MEC), Hospitais Universitários.

http://portal.mec.gov.br/hospitaisuniversitarios (acessado em 06 de maio de 2018).

6. Simões KO. Preservar para dar acesso à informação técnica no hemorio: um piloto com Procedimentos Operacionais Padrão. Especialização em Informação Científica e Tecnológica em Saúde Instituto de Comunicação e Informação Científica e Tecnológica em Saúde da Fundação Oswaldo Cruz; 2013.

7. Kitchenham B, Procedures for performing systematic reviews, Joint Technical Report Software Engineering
Group, Department of Computer Science, Keele University, United King and Empirical Software Engineering, National ICT Australia Ltd., Australia; 2004.

8. Sommerville, I. Engenharia de Software. 9. ed. Pearson PrenticeHall; 2011.

9. Peliano SV. Elaboração de diagramas da uml utilizando mapa mental. 2014 (monografia- Instituto de Ciências Exatas da Universidade Federal de Juiz de Fora). 10. Pavani Junior O, Scucuglia RI. Mapeamento e gestão por processos BPM. São Paulo: M. Books; 2011. 11. Guedes, GTA. UML 2 - Uma Abordagem Prática. 2. ed. São Paulo: Novatec; 2011. Cap. 1, 3, 4 e 10.1

RE. SAÚD. DIGI. TEC. EDU., Fortaleza, CE, v.4, n.2, p.84-98, ago./dez. 2019. ISSN: 2525-9063 


\section{Anexos}

Figura 1: Fluxo Metodológico da Revisão Integrativa de Literatura
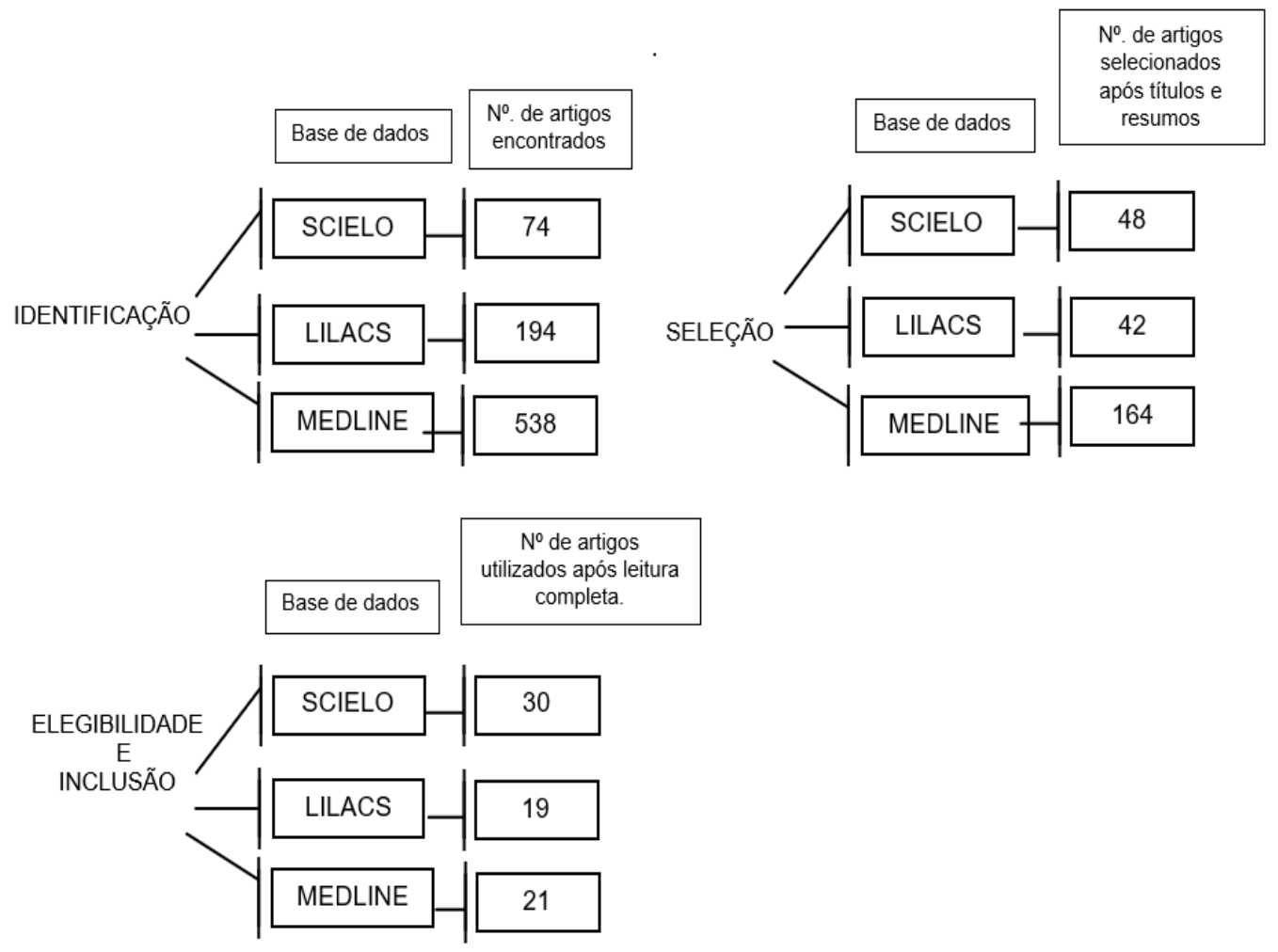

Fonte: os autores, 2018. 
Figura 2: Mapa Mental - Repositório Institucional

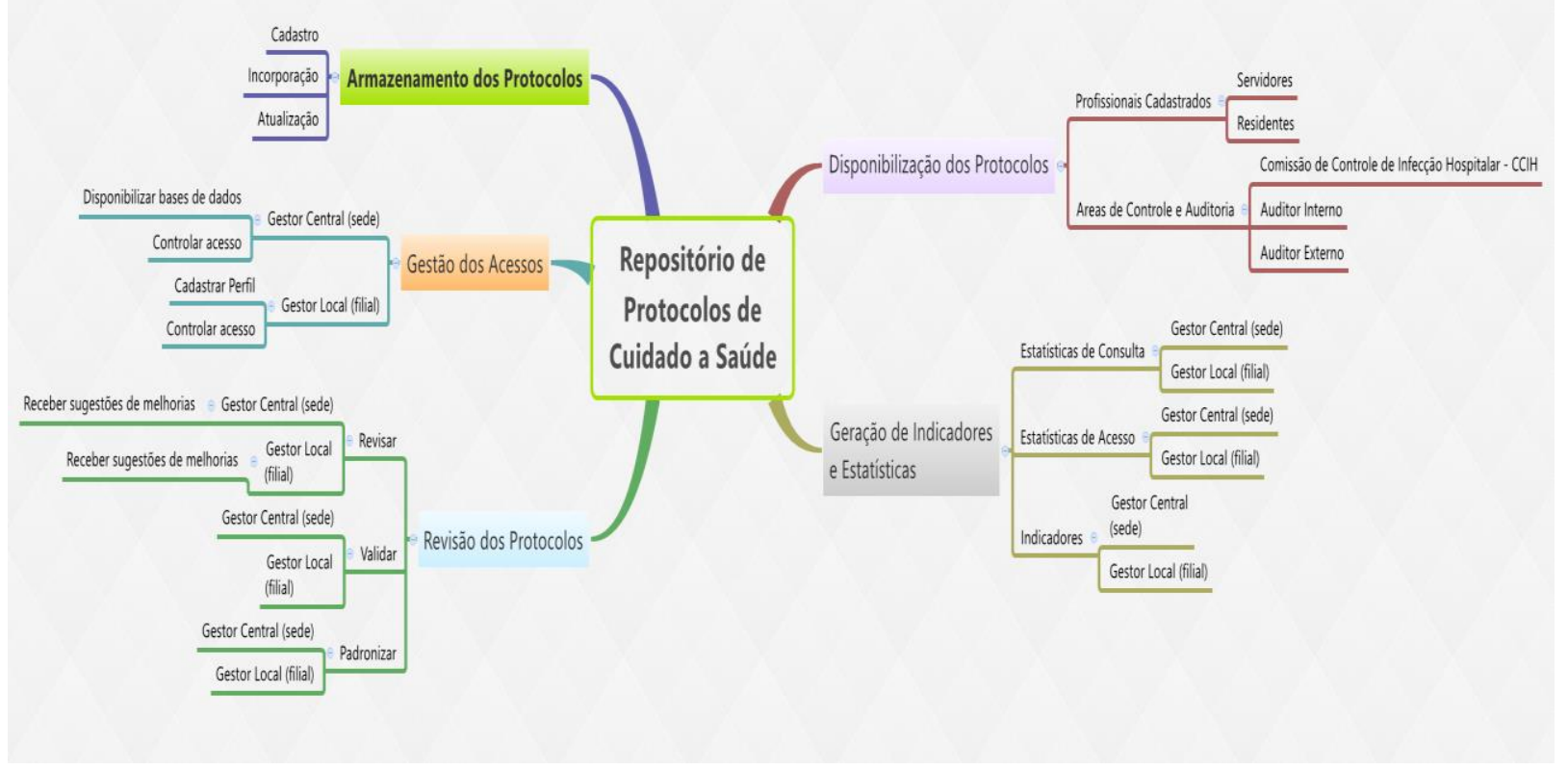

Fonte: os autores, 2018

Figura 3: Fluxograma Interfuncional dos protocolos no sistema

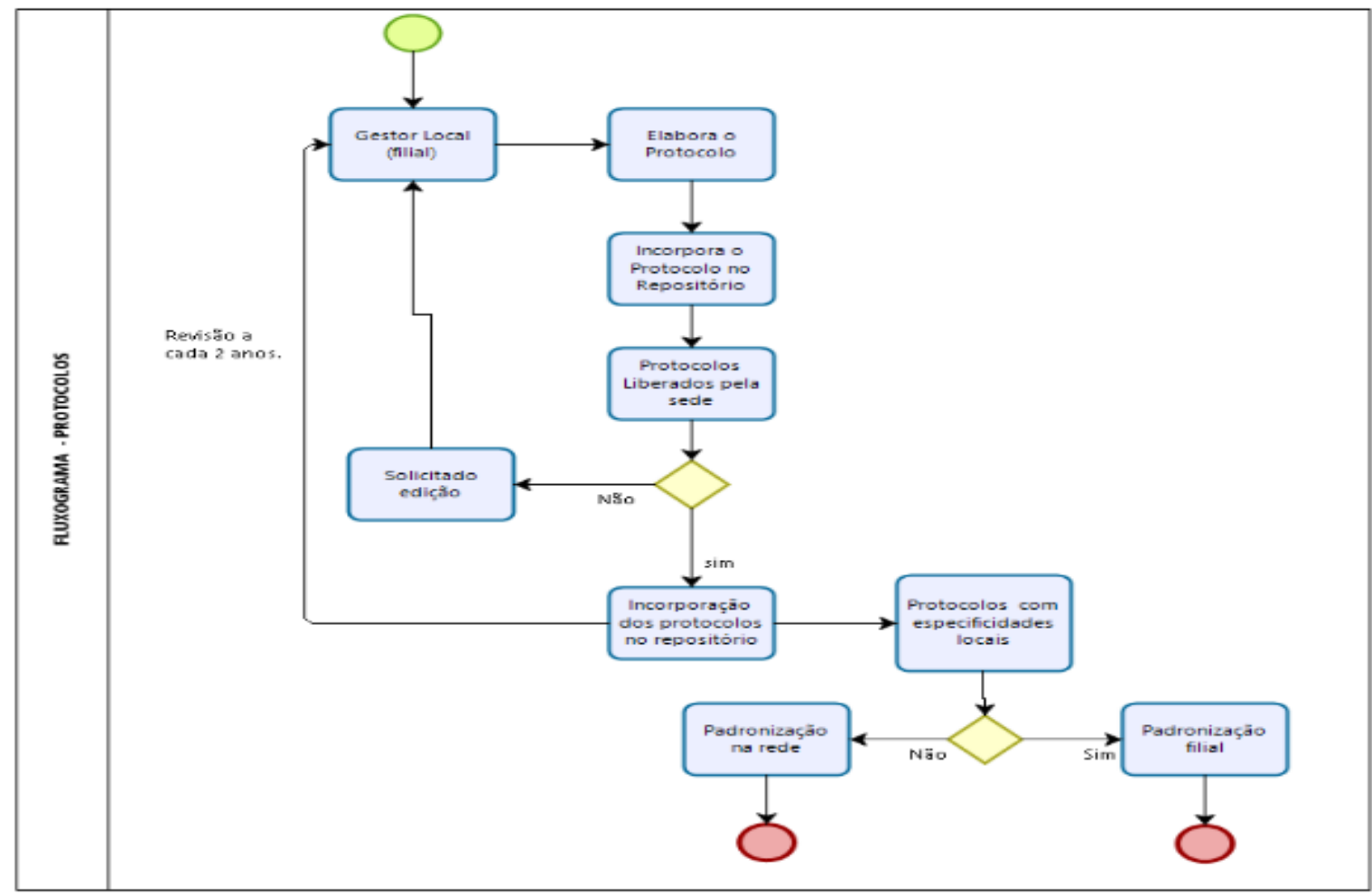

Fonte: os autores, 2018

RE. SAÚD. DIGI. TEC. EDU., Fortaleza, CE, v.4, n.2, p.84-98, ago./dez. 2019.

ISSN: 2525-9063 
Figura 4: Diagrama de Casos de Uso - Atores do sistema

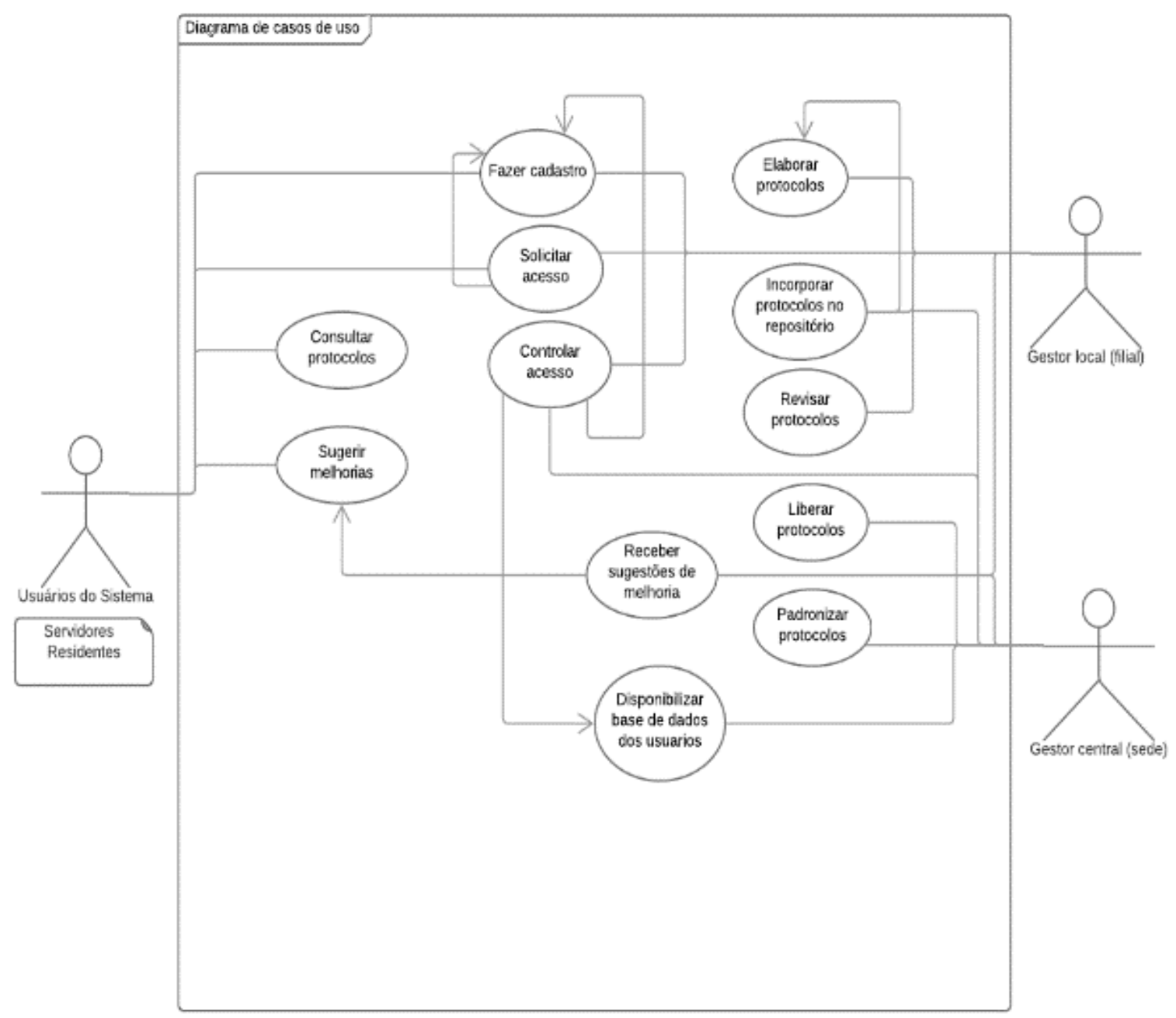

Fonte: os autores, 2018.

RE. SAÚD. DIGI. TEC. EDU., Fortaleza, CE, v.4, n.2, p.84-98, ago./dez. 2019.

ISSN: 2525-9063 
Quadro 1: Modelo padrão dos Protocolos - Informações Obrigatórias.

\begin{tabular}{|c|c|c|c|c|c|}
\hline Categoria Profissional: & $\begin{array}{l}\text { Unida } \\
\text { Elabo }\end{array}$ & $\begin{array}{l}\text { de de } \\
\text { ração }\end{array}$ & & $\mathrm{Ho}$ & pital Universitário \\
\hline \multicolumn{3}{|l|}{ Nome do protocolo } & $\begin{array}{l}\text { POP } \\
\text { Versão: }\end{array}$ & - & Páginas: \\
\hline \multicolumn{2}{|l|}{$\begin{array}{l}\text { Data de } \\
\text { elaboraçăo:__ } 1, \ldots\end{array}$} & \multicolumn{2}{|c|}{ Data de aprovaçăo: ___ ${ }^{\prime}{ }^{\prime}$} & \multicolumn{2}{|c|}{ Validade: } \\
\hline \multicolumn{2}{|c|}{ Elaborado por: (nome e cargo) } & \multicolumn{2}{|c|}{ Revisado por: (nome e cargo) } & \multicolumn{2}{|c|}{$\begin{array}{l}\text { Aprovado por: (nome e } \\
\text { cargo) }\end{array}$} \\
\hline
\end{tabular}

1- Introduçăo:

2- Definiçăo, indicaçōes e implicaçöes

3- Competência Profissional (quem deve realizar o procedimento):

4- Descriçäo dos Materiais (Se houver);

5- Descriçăo do Procedimentos (Descriçăo passo a passo):

6- Bibliografia:

7. Anexos

Fonte: os autores,2018.

RE. SAÚD. DIGI. TEC. EDU., Fortaleza, CE, v.4, n.2, p.84-98, ago./dez. 2019. ISSN: 2525-9063 


\section{Como citar este artigo}

Algeri EDBO, Costa MCMDR, Lins HWC, Silveira RS. Modelagem Descritiva de um Repositório Institucional de Protocolos de Cuidado à Saúde em hospitais universitários brasileiros. Revista de Saúde Digital e Tecnologias Educacionais. [online], volume 4, n. 2. Editor responsável: Luiz Roberto de Oliveira. Fortaleza, mês e ano, p.84-98. Disponível em: http://periodicos.ufc.br/resdite/index. Acesso em "dia/mês/ano".

Data de recebimento do artigo: 08/10/2019

Data de aprovação do artigo: 06/11/2019

RE. SAÚD. DIGI. TEC. EDU., Fortaleza, CE, v.4, n.2, p.84-98, ago./dez. 2019. 